\title{
Invariance of total positivity of a matrix under entry-wise perturbation and completion problems
}

\author{
Mohammad Adm and Jürgen Garloff
}

\begin{abstract}
A totally positive matrix is a matrix having all its minors positive. The largest amount by which the single entries of such a matrix can be perturbed without losing the property of total positivity is given. Also some completion problems for totally positive matrices are investigated.
\end{abstract}

\section{Introduction}

In this paper we consider matrices which are totally positive, i.e., all their minors are positive. For the properties of these matrices the reader is referred to the two recent monographs [5] and [11]. In the first part of our paper we are interested in the largest amount by which the single entries of such a matrix can be varied without losing the property of total positivity. This question is answered for a few specified entries in [6], see also [5, Section 9.5]. Similarly, one may ask how much the entries of a totally nonnegative matrix, i.e., a matrix having all its minors nonnegative, can be perturbed without losing the property of total nonnegativity. This problem is solved in $[2]$ for tridiagonal totally nonnegative matrices. A related question is whether all matrices lying between two totally nonnegative matrice with respect to a suitable partial ordering are totally nonnegative, too. The second author conjectured in 1982 [8] that this is true for the nonsingular totally nonnegative matrices and the so-called checkerboard ordering, see also [5, Section 3.2] and [11, Section 3.2]. In [1] we apply the so-called Cauchon algorithm, see, e.g., $[\mathbf{3}]$, to settle this conjecture.

In the second part of our paper we solve some completion problems for totally positive matrices. Here we consider the case that in a matrix some entries are specified, while the remaining ones are unspecified and are free to be chosen. All minors contained in the specified part are supposed to be positive. Then the question arises whether values for the unspecified entries can be chosen such that the resulting matrix is totally positive. Solutions of such completion problems can be found in [5, Subsections 9.1-9.4], and [10]. The starting point of our work is the recent paper $[\mathbf{9}]$.

2010 Mathematics Subject Classification. Primary 15B48.

Key words and phrases. Totally positive matrix, entry-wise perturbation, determinantal inequality, completion problem. 
The organization of our paper is as follows. In the next section we explain our notation and we collect some auxiliary results in Section 3. In Section 4 we present our main results on the perturbation of entries of totally positive matrices and we show that the derived set of determinantal conditions is minimal. In Section 5 we solve a completion problem for some new patterns of unspecified entries, hereby partially settling two conjectures in $[\mathbf{9}]$.

\section{Notation}

We now introduce the notation used in our paper. For $\kappa, n$ we denote by $Q_{\kappa, n}$ the set of all strictly increasing sequences of $\kappa$ integers chosen from $\{1,2, \ldots, n\}$. For $\alpha=\left\{\alpha_{1}, \alpha_{2}, \ldots, \alpha_{\kappa}\right\} \in Q_{\kappa, n}$ the dispersion of $\alpha$ is $d(\alpha)=\alpha_{\kappa}-\alpha_{1}-\kappa+1$. If $d(\alpha)=0$ then the index set $\alpha$ is called contiguous. Let $A$ be a real $n \times m$ matrix. For $\alpha=\left\{\alpha_{1}, \alpha_{2}, \ldots, \alpha_{\kappa}\right\} \in Q_{\kappa, n}, \beta=\left\{\beta_{1}, \beta_{2}, \ldots, \beta_{\mu}\right\} \in Q_{\mu, m}$, we denote by $A[\alpha \mid \beta]$ the $\kappa \times \mu$ submatrix of $A$ contained in the rows indexed by $\alpha_{1}, \alpha_{2}, \ldots, \alpha_{\kappa}$ and columns indexed by $\beta_{1}, \beta_{2}, \ldots, \beta_{\mu}$. We suppress the braces when we enumerate the indices explicitly. In the case that $\alpha$ or $\beta$ is obtained by taking the union of two index sets we assume that the resulting index set is ordered increasingly. When $\alpha=\beta$, the principal submatrix $A[\alpha \mid \alpha]$ is abbreviated to $A[\alpha]$. In the special case where $\alpha=\{1,2, \ldots, \kappa\}$, we refer to the principal submatrix $A[\alpha]$ as a leading principal submatrix (and to $\operatorname{det} A[\alpha]$ as a leading principal minor). By $A(\alpha \mid \beta)$ we denote the $(n-\kappa) \times(m-\mu)$ submatrix of $A$ contained in the rows indexed by the elements of $\{1,2, \ldots, n\} \backslash\left\{\alpha_{1}, \alpha_{2}, \ldots, \alpha_{\kappa}\right\}$, and columns indexed by $\{1,2, \ldots, m\} \backslash\left\{\beta_{1}, \beta_{2}, \ldots, \beta_{\mu}\right\}$ (where both sequences are ordered strictly increasingly) with the similar notation $A(\alpha)$ for the complementary principal submatrix.

A minor $\operatorname{det} A[\alpha \mid \beta]$ is called row-initial if $\alpha=\{1,2, \ldots, \kappa\}$ and $\beta \in Q_{\kappa, m}$ is contiguous, it is termed column-initial if $\alpha \in Q_{\kappa, n}$ is contiguous while $\beta=$ $\{1,2, \ldots, \kappa\}$, and initial if it is row-initial or column-initial.

The $n$-by- $n$ matrix whose only nonzero entry is a one in the $(i, j)^{t h}$ position is denoted by $E_{i j}$. We reserve throughout the notation $T_{n}=\left(t_{i j}\right)$ for the permutation matrix with $t_{i, n-i+1}=1, i=1, \ldots, n$. An $n$-by- $m$ matrix $A$ is called totally positive (abbreviated TP henceforth) and totally nonnegative (abbreviated $T N$ ) if $\operatorname{det} A[\alpha \mid \beta]>0$ and $\operatorname{det} A[\alpha \mid \beta] \geq 0$, respectively, for all $\alpha, \beta \in Q_{\kappa, n^{\prime}}, \kappa=1,2, \ldots, n^{\prime}$, and $n^{\prime}:=\min \{n, m\}$. In passing we note that if an $n$-by- $m$ matrix $A$ is $T P$ then so are its transpose and $A^{\#}:=T_{n} A T_{m}$, see, e.g., [5, Theorem 1.4.1]. We will briefly relate our results to $T P_{k}$ matrices, $k \leq n^{\prime}$. $A$ is said to be $T P_{k}$ if all its minors of order less than or equal to $k$ are positive.

We say that a rectangular array is a partial matrix if some of its entries are specified, while the remaining, unspecified, entries are free to be chosen. A partial matrix is partial TP if each of its fully specified submatrices is TP. A completion of a partial matrix is a choice of values for the unspecified entries, resulting in a matrix that agrees with the a given partial matrix in all its specified positions. A pattern $P$ is $T P$ completable if every partial $T P$ matrix with pattern $P$ has a $T P$ completion. $T P_{k}$ completion is defined analogously.

We recall from $[\mathbf{9}]$ the following definitions. We associate with a matrix the directions north, east, south, and west. So the entry in position $(1,1)$ lies north and west. We call a (possibly rectangular) pattern jagged if, whenever a position is unspecified, either all positions north and west of it are unspecified or south and east of it are, and we call a (possibly rectangular) pattern echelon if, whenever a 
position is unspecifed, either all positions north and east of it are unspecifed or south and west of it are. Either of these is referred to as single echelon, while when both occur, we say double echelon. Echelon refers to any of these possibilities.

\section{Auxiliary Results}

The following proposition shows that it suffices to consider the initial minors if one wants to check a matrix for total positivity.

Proposition 3.1. [7, Theorem 4.1], see also [5, Theorem 3.1.4] If all initial minors of a matrix $A$ are positive, then $A$ is TP.

A fundamental tool for proving in the next section inequalities between ratios of determinants is the following proposition.

Proposition 3.2. [12, Theorem 4.2] Let $\alpha, \alpha^{\prime}, \beta, \beta^{\prime}, \gamma, \gamma^{\prime}, \delta, \delta^{\prime}$ be subsets of $\{1,2, \ldots, n\}$ with $\alpha \cup \gamma=\{1,2, \ldots, p\}$ and $\alpha^{\prime} \cup \gamma^{\prime}=\left\{1,2, \ldots, p^{\prime}\right\}, q=|\alpha \cap \gamma|$, $q^{\prime}=\left|\alpha^{\prime} \cap \gamma^{\prime}\right|$, and $r:=\frac{1}{2}\left(p-q+p^{\prime}-q^{\prime}\right)$. Let $\eta$ be the unique order preserving map

$$
\eta:(\alpha \backslash \gamma) \cup(\gamma \backslash \alpha) \rightarrow\{1,2, \ldots, p-q\},
$$

and let $\eta^{\prime}$ be the unique order reversing map

$$
\eta^{\prime}:\left(\alpha^{\prime} \backslash \gamma^{\prime}\right) \cup\left(\gamma^{\prime} \backslash \alpha^{\prime}\right) \rightarrow\{p-q+1, \ldots, 2 r\} .
$$

Define the subsets $\alpha^{\prime \prime}$ and $\beta^{\prime \prime}$ of $\{1,2, \ldots, 2 r\}$ by

$$
\begin{aligned}
& \alpha^{\prime \prime}:=\eta(\alpha \backslash \gamma) \cup \eta^{\prime}\left(\gamma^{\prime} \backslash \alpha^{\prime}\right), \\
& \beta^{\prime \prime}:=\eta(\beta \backslash \delta) \cup \eta^{\prime}\left(\delta^{\prime} \backslash \beta^{\prime}\right) .
\end{aligned}
$$

Then the following two statements are equivalent:

(1) For each square $T N$ matrix $A$ of order at least $n$ the following relation holds:

$$
\operatorname{det} A\left[\alpha \mid \alpha^{\prime}\right] \operatorname{det} A\left[\gamma \mid \gamma^{\prime}\right] \leq \operatorname{det} A\left[\beta \mid \beta^{\prime}\right] \operatorname{det} A\left[\delta \mid \delta^{\prime}\right] .
$$

(2) The relations $\alpha \cup \gamma=\beta \cup \delta$ and $\alpha^{\prime} \cup \gamma^{\prime}=\beta^{\prime} \cup \delta^{\prime}$ are fulfilled and the sets $\alpha^{\prime \prime}, \beta^{\prime \prime}$ satisfy the inequality

$$
\max \left\{\left|\omega \cap \beta^{\prime \prime}\right|,\left|\omega \backslash \beta^{\prime \prime}\right|\right\} \leq \max \left\{\left|\omega \cap \alpha^{\prime \prime}\right|,\left|\omega \backslash \alpha^{\prime \prime}\right|\right\}
$$

for each subset $\omega \subseteq\{1,2, \ldots, 2 r\}$ of even cardinality.

Proposition 3.3. [13], see also [5, p. 62], [11, Theorem 2.6] The set of the $T P$-by-n matrices is dense in the class of TN $n$-by-n matrices.

The following propositions are used to solve totally positive completion problems in Section 5.

Proposition 3.4. [9, Theorem 5] Each jagged pattern is TP completable.

Proposition 3.5. [5, Theorem 9.4.4] Let $A$ be an n-by-m partial TP matrix with only one unspecified entry in the $(s, t)$ position. If $\min \{n, m\} \leq 3$, then $A$ has a TP completion. If $\min \{n, m\} \geq 4$, then any such $A$ has a TP completion if and only if $s+t \leq 4$ or $s+t \geq n+m-2$.

We remark that Proposition 3.5 is generalized in [4, Theorem 4.5] to the case that the given matrix is partial $T P_{k}, k \geq 4$. 


\section{Perturbation of Totally Positive Matrices}

In this section we consider the variation of single entries of a TP matrix $A=$ $\left(a_{i j}\right)$. For simplicity we consider here only the square case $(n=m)$. We may restrict the discussion of the off-diagonal entries to the entries which are lying above the main diagonal since the related statements for the entries below the main diagonal follow by consideration of the transposed matrix.

TheOREM 4.1. Let $A=\left(a_{i j}\right)$ be a TP matrix and $0 \leq \tau$. Then for $i \leq j$,

$$
A \pm \tau E_{i j} \text { is TP if and only if } \tau<\min S,
$$

where in each of the following eight cases $S$ is a set of ratios of minors, where the minor in the denominator is obtained from the minor in the numerator by deleting in $A$ additionally row $i$ and column $j$. If in an index sequence two indices coincide then the respective matrix has to be removed from the listing. In the following cases only the numerator matrices are listed ${ }^{1}$. The cases $(-)$ and $(+)$ refer to the -and + -sign in (4.1). In the case that $S$ is empty put $\min S:=\infty$.

(1) $i=2 m, j=2 k$

$$
\begin{aligned}
& (-) S:\left\{\begin{array}{l}
A, A(n-1, n \mid 1,2), \ldots, A(n-2 k+3, \ldots, n \mid 1, \ldots, 2 k-2), \\
A(1,2 \mid n-1, n), A(1,2,3,4 \mid n-3, n-2, n-1, n), \ldots, \\
A(1, \ldots, 2 m-2 \mid n-2 m+3, \ldots, n)
\end{array}\right. \\
& (+) S:\left\{\begin{array}{l}
A(n \mid 1), A(n-2, n-1, n \mid 1,2,3), \ldots, A(n-2 k+2, \ldots, n \mid 1, \ldots, 2 k-1), \\
A(1 \mid n), A(1,2,3 \mid n-2, n-1, n), \ldots, A(1, \ldots, 2 m-1 \mid n-2 m+2, \ldots, n)
\end{array}\right. \\
& \text { (2) } i=2 m, j=2 k+1 \\
& (-) S:\left\{\begin{array}{l}
A(n \mid 1), A(n-2, n-1, n \mid 1,2,3), \ldots, A(n-2 k+2, \ldots, n \mid 1, \ldots, 2 k-1), \\
A(1 \mid n), A(1,2,3 \mid n-2, n-1, n), \ldots, A(1, \ldots, 2 m-1 \mid n-2 m+2, \ldots, n)
\end{array}\right. \\
& (+) S:\left\{\begin{array}{l}
A, A(n-1, n \mid 1,2), \ldots, A(n-2 k+1, \ldots, n \mid 1, \ldots, 2 k), \\
A(1,2 \mid n-1, n), A(1,2,3,4 \mid n-3, n-2, n-1, n), \ldots, \\
A(1, \ldots, 2 m-2 \mid n-2 m+3, \ldots, n)
\end{array}\right. \\
& \text { (3) } i=2 m+1, j=2 k \\
& (-) S:\left\{\begin{array}{l}
A(n \mid 1), A(n-2, n-1, n \mid 1,2,3), \ldots, A(n-2 k+2, \ldots, n \mid 1, \ldots, 2 k-1), \\
A(1 \mid n), A(1,2,3 \mid n-2, n-1, n), \ldots, A(1, \ldots, 2 m-1 \mid n-2 m+2, \ldots, n)
\end{array}\right. \\
& (+) S:\left\{\begin{array}{l}
A, A(n-1, n \mid 1,2), \ldots, A(n-2 k+3, \ldots, n \mid 1, \ldots, 2 k-2), \\
A(1,2 \mid n-1, n), A(1,2,3,4 \mid n-3, n-2, n-1, n), \ldots, \\
A(1, \ldots, 2 m \mid n-2 m+1, \ldots, n)
\end{array}\right.
\end{aligned}
$$

\footnotetext{
${ }^{1}$ E.g., $A(n-1, n \mid 1,2)$ refers in case $1(-)$ to the ratio $\frac{\operatorname{det} A(n-1, n \mid 1,2)}{\operatorname{det} A(2 m, n-1, n \mid 1,2,2 k)}$.
} 
(4) $i=2 m+1, j=2 k+1$

$$
\begin{gathered}
(-) S:\left\{\begin{array}{l}
A, A(n-1, n \mid 1,2), \ldots, A(n-2 k+1, \ldots, n \mid 1, \ldots, 2 k), \\
A(1,2 \mid n-1, n), A(1,2,3,4 \mid n-3, n-2, n-1, n), \ldots, \\
A(1, \ldots, 2 m \mid n-2 m+1, \ldots, n)
\end{array}\right. \\
(+) S:\left\{\begin{array}{l}
A(n \mid 1), A(n-2, n-1, n \mid 1,2,3), \ldots, A(n-2 k+2, \ldots, n \mid 1, \ldots, 2 k-1), \\
A(1 \mid n), A(1,2,3 \mid n-2, n-1, n), \ldots, A(1, \ldots, 2 m-1 \mid n-2 m+2, \ldots, n) .
\end{array}\right.
\end{gathered}
$$

Proof. The entries in the positions $(1,1)$ and $(n, n)$ can be increased arbitrarily without loosing the property of total positivity because they enter into the top left and bottom right position, respectively, in every submatrix in which they lie. This corresponds to the fact that in the cases $1(+)$ and $4(+)$ the set $S$ is empty for $i=j=1, n$. In the remaining cases we present the proof here only for $A(\tau)=A-\tau E_{2 m, 2 k}$ (case $\left.1(-)\right)$; the proof of the other perturbations is similar. If $2 m=n$, then $2 k=n$, too. The only initial minor containing $a_{n n}-\tau$ is $\operatorname{det} A(\tau)$. By expansion of $\operatorname{det} A(\tau)$ along its bottom row we obtain

$$
\operatorname{det} A(\tau)=\operatorname{det} A-\tau \operatorname{det} A(n)
$$

from which the condition

$$
0 \leq \tau<\frac{\operatorname{det} A}{\operatorname{det} A(n)}
$$

follows. We assume now that $2 m<n$. For $\alpha \in Q_{\kappa, n}$ we set

$$
\phi(\alpha):=\frac{\operatorname{det} A(\alpha \mid \alpha)}{\operatorname{det} A(\alpha \cup\{2 m\} \mid \alpha \cup\{2 k\})} .
$$

We further use the intuitive notation

First we show the inequality

$$
\phi(0):=\frac{\operatorname{det} A}{\operatorname{det} A(2 m \mid 2 k)} .
$$

$$
\phi(0) \leq \phi(n)
$$

The inequality follows by Proposition 3.2, setting $\alpha:=\alpha^{\prime}:=\{1,2, \ldots, n\}, \beta:=$ $\beta^{\prime}:=\{1,2, \ldots, n-1\}, \gamma:=\beta \backslash\{2 m\}, \gamma^{\prime}:=\beta^{\prime} \backslash\{2 k\}, \delta:=\alpha \backslash\{2 m\}, \delta^{\prime}:=\alpha^{\prime} \backslash\{2 k\}$. Then the assumptions of Proposition 3.2 are fulfilled with $p=p^{\prime}=n, q=q^{\prime}=n-2$, and therefore $r=2, \alpha^{\prime \prime}=\{1,2\}, \beta^{\prime \prime}=\{1,3\}$. For $\omega$ the following four sets can be chosen

$$
\{1,2\},\{2,3\},\{3,4\},\{1,2,3,4\} \text {. }
$$

In all four cases the inequality (3.1) is fulfilled. Applying (4.2) to $A(n), A(n-$ $1, n), \ldots, A(2 m+1, \ldots, n)$, we obtain the chain of inequalities

$$
\phi(0) \leq \phi(n) \leq \phi(n-1, n) \leq \cdots \leq \phi(2 m+1, \ldots, n) .
$$

Now we show that all the row-initial minors of $A(\tau)$ are positive; the proof of the positivity of the column-initial minors is similar. Since by expansion of $\operatorname{det} A(\tau)$ along its $2 m^{\text {th }}$ row

$$
\operatorname{det} A(\tau)=\operatorname{det} A-\tau \operatorname{det} A(2 m \mid 2 k),
$$

we obtain the condition $\tau<\phi(0)$. Similarly for $s=0,1, \ldots, n-2 m-1$,

$$
\begin{aligned}
& \operatorname{det} A(n-s, \ldots, n)(\tau)= \\
& \operatorname{det} A(n-s, \ldots, n)-\tau \operatorname{det} A(2 m, n-s, \ldots, n \mid 2 k, n-s, \ldots, n)
\end{aligned}
$$


is positive if $\tau<\phi(n-s, \ldots, n)$. Therefore by (4.3), all leading principal minors of $A(\tau)\left(\beta_{1}=1\right)$ are positive if $\tau<\phi(0)$.

Now we consider the row-initial minors $\operatorname{det} A[\alpha \mid \beta](\tau)$, where $\beta=\left(\beta_{1}, \beta_{2}, \ldots, \beta_{s}\right)$ with $\beta_{1}>1$. If $\beta_{1}$ is odd these minors are constant or strictly increasing with respect to $\tau$ so that they remain positive under the perturbation. If $\beta_{1}$ is even, we apply the proof in the case $\beta_{1}=1$ to the submatrix $A[\alpha \mid \beta](\tau)$ and obtain the remaining conditions.

By Proposition 3.1 it follows that $A(\tau)$ is $T P$ if $\tau$ is taken as the minimum of $S$ in case $1(-)$. The necessity follows from the fact that all the initial minors are linear functions in $\tau$ and that therefore for $\min S \leq \tau$ there is an initial minor which is nonpositive.

Remark 4.2. Cases $1(-)$ and 4(-) give for $i=j=1,2$ the bound $\operatorname{det} A / \operatorname{det} A(i)$ on $\tau$; see [6, Theorems 4.2 and 4.3] and [5, Theorems 9.5.4 and 9.5.5] for related statements. In case $3(+)$ setting $i=1$ and $j=2$ we get the bound $\operatorname{det} A / \operatorname{det} A(1 \mid 2)$, see [6, Theorem 4.7] and [5, Theorem 9.5.8].

The next theorem shows that the set $S$ in Theorem 4.1 is minimal.

TheOREM 4.3. For an arbitrary TP $n$-by-n matrix $A$ the set $S$ of determinantal ratios listed in Theorem 4.1 cannot be reduced in each of the eight cases.

Proof. We present the proof only for the case 1(-); the proof of the other seven cases is similar.

It suffices to show that the following ratios are not comparable if $A$ runs over the set of the $n$-by- $n$ TP matrices

$$
\begin{aligned}
b & :=\frac{\operatorname{det} A}{\operatorname{det} A(2 m \mid 2 k)}, \\
c_{\kappa} & :=\frac{\operatorname{det} A(n-2 \kappa+3, \ldots, n \mid 1, \ldots, 2 \kappa-2)}{\operatorname{det} A(2 m, n-2 \kappa+3, \ldots, n \mid 1, \ldots, 2 \kappa-2,2 k)}, \kappa=2, \ldots, k, \\
d_{\mu} & :=\frac{\operatorname{det} A(1, \ldots, 2 \mu-2 \mid n-2 \mu+3, \ldots, n)}{\operatorname{det} A(1, \ldots, 2 \mu-2,2 m \mid 2 k, n-2 \mu+3, \ldots, n)}, \quad \mu=2, \ldots, m .
\end{aligned}
$$

We show here only that the ratios $c_{\kappa}$ and $d_{\mu}$ are not comparable; the proof of the other cases is similar (and easier). We first prove that the inequality $c_{\kappa} \leq d_{\mu}$ does not hold for all TP $n$-by- $n$ matrices $A$. To apply Proposition 3.2, we choose

$$
\begin{aligned}
\alpha & :=\{1, \ldots, n-2 \kappa+2\}, \alpha^{\prime}:=\{2 \kappa-1, \ldots, n\}, \\
\delta & :=\{2 \mu-1, \ldots, n\}, \delta^{\prime}:=\{1, \ldots, n-2 \mu+2\}, \\
\beta & :=\alpha \backslash\{2 m\}, \beta^{\prime}=\alpha^{\prime} \backslash\{2 k\}, \\
\gamma & :=\delta \backslash\{2 m\}, \gamma^{\prime}=\delta^{\prime} \backslash\{2 k\} .
\end{aligned}
$$

Then we have

$$
\alpha \cup \gamma=\beta \cup \delta=\alpha^{\prime} \cup \gamma^{\prime}=\beta^{\prime} \cup \delta^{\prime}
$$

by

$$
\begin{aligned}
& 2 \mu-1<2 m \leq n-2 \kappa+2, \\
& 2 \kappa-1<2 k \leq n-2 \mu+2,
\end{aligned}
$$

hence $p=p^{\prime}=n$;

$$
\begin{aligned}
\alpha \cap \gamma & =\{2 \mu-1, \ldots, n-2 \kappa+2\} \backslash\{2 m\}, \\
\alpha^{\prime} \cap \gamma^{\prime} & =\{2 \kappa-1, \ldots, n-2 \mu+2\} \backslash\{2 k\},
\end{aligned}
$$


hence $q=q^{\prime}=n-2 \kappa-2 \mu+3$;

$$
\begin{aligned}
\eta:\{1, \ldots, 2 \mu-2,2 m\} \cup\{n-2 \kappa+3, \ldots, n\} \rightarrow\{1, \ldots, 2 \kappa+2 \mu-3\}, \\
\eta^{\prime}:\{2 k, n-2 \mu+3, \ldots, n\} \cup\{1, \ldots, 2 \kappa-2\} \rightarrow\{2 \kappa+2 \mu-2, \ldots, 4 \kappa+4 \mu-6\}, \\
\alpha^{\prime \prime}=\eta(\{1, \ldots, 2 \mu-2,2 m\}) \cup \eta^{\prime}(\{1, \ldots, 2 \kappa-2\}) \\
=\{1, \ldots, 2 \mu-1\} \cup\{2 \kappa+4 \mu-3, \ldots, 4 \kappa+4 \mu-6\}, \\
\beta^{\prime \prime}=\eta(\{1, \ldots, 2 \mu-2\}) \cup \eta^{\prime}(\{1, \ldots, 2 \kappa-2,2 k\}) \\
=\{1, \ldots, 2 \mu-2\} \cup\{2 \kappa+4 \mu-4, \ldots, 4 \kappa+4 \mu-6\} .
\end{aligned}
$$

Let $w:=\{2 \kappa+4 \mu-4,2 \kappa+4 \mu-3\}$. Then the inequality (3.1) is not fulfilled and by Proposition 3.2 there exists a $T N$ matrix $A_{1}$ for which the inequality $c_{\kappa}>d_{\mu}$ holds. By interchanging the role of sets $\alpha, \alpha^{\prime}, \gamma, \gamma^{\prime}$ with the sets $\beta, \beta^{\prime}, \delta, \delta^{\prime}$, we find by Proposition 3.2 (choosing $w:=\{2 \mu-2,2 \mu-1\}$ ) a $T N$ matrix $A_{2}$ for which the inequality $c_{\kappa}<d_{\mu}$ holds. So the ratios $c_{\kappa}$ and $d_{\mu}$ are not comparable on the set of the $T N$ matrices. By using Proposition 3.3 we find two TP matrices satisfying the respective inequalities which shows that also on the set of TP matrices the ratios $c_{\kappa}$ and $d_{\mu}$ are not comparable.

ExAmPLE 4.4. Let $A$ be the Pascal matrix of order 4, i.e.,

$$
A=\left(\begin{array}{cccc}
1 & 1 & 1 & 1 \\
1 & 2 & 3 & 4 \\
1 & 3 & 6 & 10 \\
1 & 4 & 10 & 20
\end{array}\right)
$$

Then $A$ is $T P$, see, e.g., [5, Example 0.1.6]. In Table 1 we give the largest interval from which $\tau$ can be chosen according to Theorem 4.1 such that the matrix $A(\tau):=A+\tau E_{i j}$ is $T P, i, j=1, \ldots, n, i \leq j$. The intervals are given in the $(i, j)$ position of the respective entry. In each case, if $\tau$ is chosen as an endpoint of the interval, the respective matrix $A(\tau)$ contains a vanishing minor.

$$
\begin{aligned}
& \left(-\frac{1}{4}, \infty\right) \quad\left(-\frac{1}{6}, \frac{1}{6}\right) \quad\left(-\frac{1}{4}, \frac{1}{8}\right) \quad\left(-\frac{1}{3}, \frac{1}{3}\right) \\
& \left(-\frac{1}{14}, \frac{1}{4}\right) \quad\left(-\frac{1}{7}, \frac{1}{11}\right) \quad\left(-\frac{1}{3}, \frac{1}{3}\right) \\
& \left(-\frac{1}{10}, \frac{1}{2}\right) \quad\left(-1, \frac{1}{3}\right) \\
& (-1, \infty)
\end{aligned}
$$

TABLE 1. The largest perturbation intervals in Example 4.4.

\section{TP Completion Problems}

In this section we consider $T P$ completion problems for some new patterns of the unspecified entries.

We recall from $[\mathbf{9}]$ the definition of the patterns $P_{1}, P_{1}{ }^{\prime}$, and $P_{2}$. Let $A$ be an $n$-by- $m$ matrix. We say that $A$ has a $P_{1}$ or $P_{1}^{\prime}$ pattern if $A$ has just one unspecified entry, viz. in the $(1, m)$ or $(n, 1)$ positions, respectively. The $P_{2}$ pattern has just 
two unspecified entries, viz. in positions $(1, m)$ and $(n, 1)$. Since by Proposition 3.1 a matrix is $T P$ if and only if its initial minors are positive, a partial $T P$ matrix with a $P_{1}$ pattern has a $T P$ completion if and only if the upper right entry can be chosen so that the upper right minors with contiguous index sets are all positive. A similar condition holds for a $P_{1}^{\prime}$ pattern. We introduce two further patterns. We say that $A$ has a $P_{3}$ pattern if $3 \leq m$ and the unspecified entries are $a_{i j}$,

$$
\begin{aligned}
& i=1, \ldots, l, j=3, \ldots, k, \text { and } i=r, \ldots, n, j=1, \ldots, m-2, \\
& \text { with } l \in\{1, \ldots, n-1\}, r \in\{l+1, \ldots, n\} .
\end{aligned}
$$

$A$ has a $P_{4}$ pattern if $2 \leq n, 4 \leq m$ and the unspecified entries are $a_{i j}$,

$$
i=1, \ldots, l, j=1, \ldots, k, \text { and } i=1, \ldots, r, j=k+3, \ldots, m \text {, and } i=
$$$$
p, \ldots, n, j=1, \ldots, h \text {, and } i=t, \ldots, n, j=h+3, \ldots, m \text {, }
$$$$
\text { with } 1 \leq r \leq l<t \leq p \leq n, h, k \in\{1, \ldots, m-3\} \text {. }
$$

Examples 1 and 2 in [9] and 9.1.1 in [5] show that the $P_{1}, P_{1}^{\prime}$, and $P_{2}$ patterns are not $T P$ completable if $4 \leq \min \{n, m\}$. This explains why in the sequel the index ranges will often start at 3 .

THEOREM 5.1. Let $A$ be an $n$-by-m partial TP matrix with the unspecified entries $a_{i j}, i=1, \ldots, l, j=3, \ldots, k$, where $l \leq n, k \leq m$. Then $A$ is TP completable.

Proof. Let $B_{l k}:=A[l, \ldots, n \mid 1,2, k, \ldots, m]$. Then by Proposition $3.5, B_{l k}$ is $T P$ completable. We enter the value for the unspecified entry $a_{l k}$ into the matrix $A$ and call the resulting matrix $A_{l k}$. If $l>1$ let $B_{l-1, k}:=A_{l k}[l-1, \ldots, n \mid$ $1,2, k, \ldots, m]$. Then by Proposition $3.5, B_{l-1, k}$ is TP completable and similarly as for the entry $a_{l k}$ we obtain the $n$-by-m partial TP matrix $A_{l-1, k}$ which has one unspecified entry less than $A_{l k}$. Now we continue in this manner until we find values for all the unspecified entries in column $k$ resulting in the partial TP matrix $A_{1 k}$. If $k>3$ repeat the above process with the partial TP matrix $A_{1 k}$ to find values for the unspecified entries in the columns $k-1, \ldots, 3$. At the end of this process we get the matrix $A_{13}$ which is $T P$.

COROLlary 5.2. Let $A$ be an $n$-by-m partial TP matrix with the unspecified entries $a_{i j}, i=r, \ldots, n, j=k, \ldots, m-2$, where $r \leq n, k \leq m-2$. Then $A$ is TP completable.

Proof. The matrix $A^{\#}$ is a partial TP matrix with the same pattern of unspecified entries as the one considered in Theorem 5.1, whence $A^{\#}$ is TP completable. Then $A$ is $T P$ completable, too.

By application of Theorem 5.1 and Corollary 5.2 to $A, A^{\#}$, or $A^{T}$ it follows that a partial TP matrix $A$ whose pattern is a single echelon pattern is $T P$ completable if and only if it contains no $P_{1}$ or $P_{1}^{\prime}$ as a subpattern. This settles $[\mathbf{9}$, Conjecture 1] in a special case.

THEOREM 5.3. Let $3 \leq m$ and $A$ be an $n$-by-m partial TP matrix with the unspecified entries $a_{1 j}, j=3, \ldots, m$, and $a_{i 1}, i=l, \ldots, n$, with $l \leq 4$. Then $A$ is TP completable.

Proof. If $l=1$ or 2 then it is easy to find values for the unspecified entries in the positions $(1,1)$ and $(2,1)$, respectively, so let $l \in\{3,4\}$. Let $B_{1}:=A[2, \ldots, n \mid$ $1, \ldots, m]$. Then by taking the transpose of $B_{1}$ and using Theorem 5.1 (with $m$ 
replaced by $n), B_{1}$ is $T P$ completable. We enter the values for the unspecified entries $a_{i 1}, i=l, \ldots, n$, into the matrix $A$ and call the resulting matrix $A_{1}$. The matrix $A_{1}$ is a partial TP matrix with the unspecified entries in the first row. By Theorem $5.1 A_{1}$ is TP completable, and so $A$ is TP completable.

TheOREm 5.4. Let $3 \leq m, 4 \leq n$, and $A$ be an $n$-by-m partial TP matrix with the unspecified entries $a_{i j}, i=1, \ldots, l, j=3, \ldots, m$, and $i=l+3, \ldots, n$, $j=1, \ldots, h$, where $h \leq m$. Then $A$ is TP completable.

Proof. Let $B_{h}:=[l+1, \ldots, n \mid h, \ldots, m]$. Then $B_{h}$ is a partial TP matrix and by Theorem 5.1 (taking the transpose), $B_{h}$ is TP completable. We enter the values for the unspecified entries of $B_{h}$ into the matrix $A$ and call the resulting matrix $A_{h}$. Repeat the last step to find values for the unspecified entries in the lower part of the columns $h-1, \ldots, 2$. At the end of this process we get the partial TP matrix $A_{2}$ having the unspecified entries in the upper right corner and in the first column below the position $(1, l+3)$. To find values for the unspecified entries in the first column, we proceed analogously to the proof of Theorem 5.3. Let $C:=A_{2}[l+1, \ldots, n \mid 1, \ldots, m]$. Then by Theorem $5.1 C$ is $T P$ completable. Since only the initial minors of $A$ need to be positive, $C$ can be completed independently of the entries of $A[1, \ldots, l \mid 1,2]$. We enter the values for the unspecified entries of the first column into the matrix $A_{2}$ and call the resulting matrix $A_{1}$; then $A_{1}$ is a partial TP matrix with the unspecified entries in the upper right corner. We apply Theorem 5.1 on $A_{1}$ and it follows that $A_{1}$ is $T P$ completable. Therefore $A$ is $T P$ completable.

The following theorem combines the patterns of Theorem 5.1 and Corollary 5.2 and is related to Proposition 3.4.

THEOREM 5.5. If $A=\left(a_{i j}\right)$ is an $n$-by-m partial TP matrix with a $P_{3}$ pattern, then $A$ is TP completable.

Proof. Let $A$ be an $n$-by- $m$ partial $T P$ matrix with a $P_{3}$ pattern. We distinguish two cases.

Case $(1): k=m$. Let $B_{1}:=A[l+1, \ldots, n \mid 1, m-1, m]$. Then by applying Proposition 3.5 successively to $C_{\rho}:=B_{1}[l+1, \ldots, \rho \mid 1, m-1, m]$ we find values for the unspecified entries $a_{\rho 1}, \rho=r, \ldots, n$. Therefore $B_{1}$ is $T P$ completable. We enter the values for the unspecified entries of $B_{1}$ into the matrix $A$ and call the resulting matrix $A_{1}$. To find values for the unspecified entries in the second column of $A_{1}$ in and below position $(r, 2)$ we similarly apply Proposition 3.5 to the submatrix $B_{2}:=A_{1}[l+1, \ldots, n \mid 1,2, m-1, m]$. This completion can be accomplished independently of the entries of $A[1, \ldots, l \mid 1,2]$, see the proof of Theorem 5.4. We enter the values for the unspecified entries of $B_{2}$ into the matrix $A$ and call the resulting matrix $A_{2}$. Let $B_{3}:=A_{2}[l+1, \ldots, n \mid 1,2, \ldots, m]$. Then $B_{3}$ is partial $T P$ and by Corollary 5.2 TP completable. We enter the values for the unspecified entries of $B_{3}$ into the matrix $A_{2}$ and call the resulting matrix $A_{3}$. By Theorem $5.1 A_{3}$ is TP completable and hence $A$ is $T P$ completable.

Case (2): $k<m$. We consider first the case $k=m-1$. If $l+1=r$, then we can choose a positive number for the unspecified entry $a_{l, m-1}$ such that the matrix remains partial $T P$. If $l+1<r$, let $B_{l, m-1}:=A[l, \ldots, r-1 \mid 1,2, m-1, m]$ and $B_{l, m-1}^{\prime}:=A[l, \ldots, n \mid m-1, m]$. Then by Proposition 3.5 both submatrices are $T P$ completable. Moreover, we can choose in both matrices a common value for the 
unspecified entry $a_{l, m-1}$ because the only nontrivial initial minor of the submatrix $B_{l, m-1}^{\prime}$ containing this entry, viz. $\operatorname{det} B_{l, m-1}^{\prime}[l, l+1 \mid m-1, m]$, is also an initial minor of the submatrix $B_{l, m-1}$.

We enter the value for the unspecified entry $a_{l, m-1}$ into the matrix $A$ and call the resulting matrix $A_{l, m-1}$. Repeating this process we find values for the unspecified entries in column $m-1$ and finally obtain the partial TP matrix $A_{1, m-1}$. Let $C:=A_{1, m-1}[1, \ldots, r-1 \mid 1, \ldots, m]$. By Theorem 5.1, $C$ is TP completable. We enter the values for the unspecified entries in $A_{1, m-1}$ and call the resulting matrix $A^{\prime}$, which is a partial TP matrix. By Corollary 5.2, $A^{\prime}$ is $T P$ completable and we can conclude that $A$ is also TP completable.

If $k<m-1$ we follow the proof in the case $k=m-1$ but we may end the proof already with the definition of the matrix $C$.

THEOREM 5.6. If $A=\left(a_{i j}\right)$ is an $n-b y-m$ partial TP matrix with a $P_{4}$ pattern, then $A$ is TP completable.

Proof. Let A be an $n$-by-m partial TP matrix with a $P_{4}$ pattern. Without loss of generality we may assume that $l=r$ and $p=t$. Otherwise let $B_{0}:=$ $A[r+1, \ldots, p-1 \mid 1, \ldots, m]$. Then $B_{0}$ has a jagged pattern and can be TP completed by Proposition 3.4 (independently of the entries of $A[1, \ldots, r \mid k+1, k+2]$ and $A[p, \ldots, n \mid h+1, h+2])$. In what follows we therefore assume that $l=r$ and $p=t$. Let $B_{1}:=A[1, \ldots, p-1 \mid k+1, \ldots, m]$; then $B_{1}$ is $T P$ completable by Theorem 5.1. We enter the values for the unspecified entries of $B_{1}$ into the matrix $A[1, \ldots, p-1 \mid 1, \ldots, m]$ and call the resulting matrix $B_{2}$. Since $B_{2}$ is a partial TP matrix with a jagged pattern it is $T P$ completable by Proposition 3.4. We enter the values for the unspecified entries of $B_{2}$ into the matrix $A$ and call the resulting matrix $A_{1}$. Proceeding with $A_{1}^{\#}$ we obtain similarly the TP completion of $A_{1}$ and in this way of $A$, too.

THEOREM 5.7. If $A=\left(a_{i j}\right)$ is an $n$-by-m partial TP matrix with the unspecified entries $a_{i j} i=1, \ldots, l, j=1, \ldots, k$, and $i=1, \ldots, r, j=k+3, \ldots, m$, and $i=t, \ldots, n, j=1, \ldots, m-2$, with $r \leq l<t$, then $A$ is TP completable.

Proof. Without loss of generality we may assume that $r=l$, see the proof of Theorem 5.6. Let $C_{1}:=A[r+1, \ldots, n \mid 1, \ldots, k, m-1, m]$. Then $C_{1}$ is $T P$ completable by Corollary 5.2. We enter the values for the unspecified entries of $C_{1}$ into the matrix $A$ and call the resulting matrix $A_{1}$. Let $C_{2}:=A_{1}[r+1, \ldots, n \mid$ $1, \ldots, k+1, m-1, m]$. Then by an argument similar to that used in the proof of Theorem 5.1, by starting from the unspecified entry $a_{t, k+1}$, and proceeding downwards, we can find values for the unspecified entries in $C_{2}$; so $C_{2}$ is $T P$ completable. We enter the values for the unspecified entries of $C_{2}$ into the matrix $A_{1}$ and call the resulting matrix $A_{2}$. Similarly, we can find values for the unspecified entries in the column $k+2$, then we enter these values into the matrix $A_{2}$ and call the resulting matrix $A_{3}$. Let $D:=A_{3}[r+1, \ldots, n \mid 1, \ldots, m]$; then $D$ is a partial $T P$ matrix with the same type of pattern as the one treated in Corollary 5.2 , thus $D$ is $T P$ completable. We enter the values for the unspecified entries in $D$ into the matrix $A_{3}$ and call the resulting matrix $A_{4}$, where $A_{4}$ is a partial TP matrix. Let $D_{1}:=A_{4}[1, \ldots, n \mid k+1, \ldots, m]$; then $D_{1}$ is $T P$ completable by Theorem 5.1 (with $k=m$ ). We enter the values for the unspecified entries of $D_{1}$ into $A_{4}$ and call the resulting matrix $A_{5}$ which is partial TP. Since $A_{5}$ has a jagged pattern, it is completable by Proposition 3.4 , whence $A$ is $T P$ completable. 
THEOREM 5.8. If $A=\left(a_{i j}\right)$ is an $n$-by-m partial TP matrix with the unspecified entries $a_{i j} i=1, \ldots, l, j=1, \ldots, k$, and $i=1, \ldots, r, j=k+3, \ldots, m$, and $i=t, \ldots, n, j=h, \ldots, m$, with $r \leq l<t$, and $h<k$, then $A$ is TP completable.

Proof. We may assume without loss of generality that $r=l$. Otherwise we proceed as follows: Let $B_{1}:=A[r+1, \ldots, t-1 \mid h, \ldots, m]$. Then $B_{1}$ is partial $T P$ with a jagged pattern, thus $T P$ completable. If $h>1$ we have to take into account the entries of $A[t, \ldots, n \mid 1, \ldots, h-1]$ when we want to extend the completion to the left. We proceed element-wise by taking successively the entries $a_{l, h-1}$, $a_{l-1, h-1}, \ldots, a_{r+1, h-1}, a_{l, h-2}, \ldots, a_{r+1, h-2}, \ldots, a_{r+1,1}$. For a fixed entry we consider the submatrices which have the chosen entry as the only unspecified entry, viz in position $(1,1)$. For each such submatrix we can find a positive number such that the matrix is TP. Then we take the maximum of all these positive numbers (for the chosen entry).

The matrix $C_{1}:=A[r+1, \ldots, n \mid 1, \ldots, k+1]$ is a partial $T P$ matrix with a jagged pattern and by Proposition $3.4 C_{1}$ is $T P$ completable. We enter the values for the unspecified entries of $C_{1}$ into the matrix $A$ and call the resulting matrix $A_{1}$. Let $C_{2}:=A_{1}[r+1, \ldots, n \mid 1, \ldots, k+2]$, and $C_{2}^{\prime}:=A_{1}[1, \ldots, n \mid k+1, k+2]$. Then both submatrices are partial TP matrices with jagged patterns, and so by the argument used in the proof of Theorem 5.5, Case (2), common values for the unspecified entries can be found. We enter the values for the unspecified entries of $C_{2}$ into the matrix $A_{1}$ and call the resulting matrix $A_{2}$. Let $C_{3}:=A_{2}[r+1, \ldots, n \mid 1, \ldots, m]$. Then $C_{3}$ is a partial TP matrix with a jagged pattern, thus $C_{3}$ is TP completable. We enter the values for the unspecified entries of $C_{3}$ into the matrix $A_{2}$ and call the resulting matrix $A_{3}$. Since $A_{3}$ is a partial TP matrix with the same type of pattern as the one considered in the proof of Theorem 5.6, $A_{3}$ is TP completable. Hence $A$ is $T P$ completable.

Theorems 5.7 and 5.8 represent certain instances of a jagged echelon pattern with no $P_{1}, P_{1}^{\prime}$, or $P_{2}$ as a subpattern. Thus both theorems and the following remark settle [9, Conjecture 2] in some special cases leaving this conjecture unresolved only in the case of a double echelon pattern.

REMARK 5.9. The following patterns can be proven to be $T P$ completable by using similar methods as in the proofs of the Theorems 5.6, 5.7, and 5.8.

The entries $a_{i j}$ are unspecified for

(1) $i=1, \ldots, l, j=1, \ldots, k$, and $i=r, \ldots, n, j=1, \ldots, m-2$, with $l<r$;

(2) $i=1, \ldots, l, j=3, \ldots, m$, and $i=r, \ldots, n, j=k, \ldots, m$, with $l<r$;

(3) $i=1, \ldots, l, j=3, \ldots, m$, and $i=r, \ldots, n, j=1, \ldots, k$, and $i=t, \ldots, n$, $j=k+3, \ldots, m$, with $l<t<r$

(4) $i=1, \ldots, l, j=1, \ldots, k$, and $i=r, \ldots, n, j=1, \ldots, h$, and $i=t, \ldots, n$, $j=h+3, \ldots, m$, with $l<t<r$ and $h<k$.

Remark 5.10. By using [4, Theorem 5.4] instead of Proposition 3.5 all the results of Section 5 carry over to the case that the given matrix $A$ is partial $T P_{k}$, $k \geq 4$.

\section{References}

[1] Mohammad Adm and Jürgen Garloff, Intervals of totally nonnegative matrices, Linear Algebra Appl. 439 (2013), no. 12, 3796-3806, DOI 10.1016/j.laa.2013.10.021. MR3133459 
[2] Mohammad Adm and Jürgen Garloff, Invariance of total nonnegativity of a tridiagonal matrix under element-wise perturbation, Oper. Matrices 8 (2014), no. 1, 129-137, DOI 10.7153/oam-08-06. MR3202931

[3] Mohammad Adm and Jürgen Garloff, Improved tests and characterizations of totally nonnegative matrices, Electron. J. Linear Algebra 27 (2014), 588-610. MR3266168

[4] V. Akin, Charles R. Johnson, and Shahla Nasserasr, $\mathrm{TP}_{K}$ completion of partial matrices with one unspecified entry, Electron. J. Linear Algebra 27 (2014), 426-443. MR3240024

[5] Shaun M. Fallat and Charles R. Johnson, Totally nonnegative matrices, Princeton Series in Applied Mathematics, Princeton University Press, Princeton, NJ, 2011. MR2791531 (2012d:15001)

[6] Shaun M. Fallat, Charles R. Johnson, and Ronald L. Smith, The general totally positive matrix completion problem with few unspecified entries, Electron. J. Linear Algebra 7 (2000), 1-20. MR1737244 (2000k:15044)

[7] Mariano Gasca and Juan Manuel Peña, Total positivity and Neville elimination, Linear Algebra Appl. 165 (1992), 25-44, DOI 10.1016/0024-3795(92)90226-Z. MR1149743 (93d:15031)

[8] Jürgen Garloff, Criteria for sign regularity of sets of matrices, Linear Algebra Appl. 44 (1982), 153-160, DOI 10.1016/0024-3795(82)90010-6. MR657704 (84f:15024)

[9] Charles R. Johnson and Zhen Wei, Asymmetric TP and TN completion problems, Linear Algebra Appl. 438 (2013), no. 5, 2127-2135, DOI 10.1016/j.laa.2012.11.008. MR3005280

[10] Cristina Jordán and Juan R. Torregrosa, The totally positive completion problem, Linear Algebra Appl. 393 (2004), 259-274, DOI 10.1016/j.laa.2004.03.015. MR2098618 (2005h:15065)

[11] Allan Pinkus, Totally positive matrices, Cambridge Tracts in Mathematics, vol. 181, Cambridge University Press, Cambridge, 2010. MR2584277 (2010k:15065)

[12] Mark Skandera, Inequalities in products of minors of totally nonnegative matrices, J. Algebraic Combin. 20 (2004), no. 2, 195-211, DOI 10.1023/B:JACO.0000047282.21753.ae. MR2104676 (2005k:15046)

[13] Anne M. Whitney, A reduction theorem for totally positive matrices (English, with Hebrew summary), J. Analyse Math. 2 (1952), 88-92. MR0053173 (14,732c) 